TU-576

\title{
Pattern of Neutrino Oscillations in Supersymmetry with Bilinear R-parity Violation
}

\author{
Fumihiro Takayama* and Masahiro Yamaguchi ${ }^{\dagger}$ \\ Department of Physics, Tohoku University \\ Sendai 980-8578, Japan
}

(October 1999)

\begin{abstract}
Neutrino masses and their mixing are studied in detail in the framework of a supersymmetric standard model with bilinear R-parity violation. In this scenario, the mixing matrix among the neutrinos is in a restrictive form. We find that only the small angle MSW solution is allowed for the solar neutrino problem when the results of the $\mathrm{CHOOZ}$ experiment are combined with the mass squared difference and mixing angle suggested by the atmospheric neutrino data. Collider signals of this scenario are also discussed.
\end{abstract}

*e-mail: takayama@tuhep.phys.tohoku.ac.jp

†e-mail: yama@tuhep.phys.tohoku.ac.jp 
Neutrino masses and their mixing are an intriguing window to physics beyond the Standard Model. Neutrino oscillations among massive neutrinos have been strongly suggested by the atmospheric neutrino data at Super-Kamiokande [1] as well as by the solar neutrino problem [2].

Here we shall consider this issue in the framework of a supersymmetric (SUSY) extension of the standard model [3]. Usually in the supersymmetric standard model, one imposes Rparity conservation to avoid too fast proton decay. However, it is well known that the stability of the proton does not forbid all R-parity non-conservation, but allows R-parity violation such that either lepton number or baryon number is broken, because the nucleon decays normally require not only the baryon number violation but also the lepton number violation.

Actually it is widely recognized that the R-parity violation with the lepton number non-conservation [4.5] is a promising approach to give neutrino masses and mixing without introducing right-handed neutrinos [6]. Here we shall consider the case where the R-parity is broken only by bilinear terms [5]. This is particularly interesting because it is compatible with the idea of grand unified theory (GUT) where quarks and leptons typically belong to one representation of a GUT group.

An important feature of this mechanism of generating neutrino masses is that one may be able to see the R-parity violation in future collider experiments [0],8].

In this paper, we shall investigate the nature of the neutrino mass matrix in this scenario. We find that large angle solutions to the solar neutrino deficit are not allowed when we combine the CHOOZ result [9] with the $\nu_{\tau}-\nu_{\mu}$ solution of the atmospheric neutrino problem. This singles out the small mixing MSW solution [11] for the solar neutrino in this scenario. We will then show that the soft SUSY breaking masses should be finely adjusted to simultaneously explain the atmospheric neutrino anomaly and the solar neutrino problem. We will also discuss implications of this specific pattern of the R-parity violation at collider experiments.

Let us first describe the model we are considering. The particle contents of the model 
are the same as those of the minimal supersymmetric standard model (MSSM). R-parity breaking bilinear terms are assumed to exist in superpotential

$$
W=\mu H_{D} H_{U}+\mu_{i} L_{i} H_{U}+Y_{i}^{L} L_{i} H_{D} E_{i}^{c}+Y_{i}^{D} Q_{i} H_{D} D_{i}^{c}+Y_{i j}^{U} Q_{i} H_{U} U_{j}^{c} .
$$

Here $H_{D}, H_{U}$ are two Higgs doublets, $L_{i}$ a $S U(2)_{L}$ doublet lepton, $E_{i}^{c}$ is a singlet lepton, $Q_{i}$ a doublet quark, $U_{i}^{c}, D_{i}^{c}$ a singlet quark of up and down type, respectively. Suffices $i, j$ stand for generations. In this basis, the Yukawa coupling matrices for the charged leptons and for the down-type quarks are assumed to be diagonal with respect to the generation indices. The soft SUSY breaking terms in the scalar potential are taken to be of the form

$V^{\mathrm{soft}}=B H_{D} H_{U}+B_{i} \tilde{L}_{i} H_{U}+m_{H_{D}}^{2} H_{D} H_{D}^{\dagger}+m_{H_{U}}^{2} H_{U} H_{U}^{\dagger}+m_{H L_{i}}^{2} \tilde{L}_{i} H_{D}^{\dagger}+m_{L_{i j}}^{2} \tilde{L}_{i} \tilde{L}_{j}^{\dagger}+\cdots$

where we have written only bilinear terms explicitly. In this paper, we assume lepton-Higgs universality in the soft masses, namely

$$
B_{i} / B=\mu_{i} / \mu, m_{L_{i} j}^{2}=\delta_{i j} m_{L_{i}}^{2}, m_{L_{i}}^{2}=m_{H_{D}}^{2}, m_{H L_{i}}^{2}=0
$$

These conditions are achieved, e.g. in the minimal supergravity t or in scenarios of gauge mediated supersymmetry breaking (GMSB). Our motivation to consider this universality is alignment of vacuum expectation values with the lepton number violating bilinear terms [14]. We shall return to this point later.

Of course, the lepton-Higgs universality is not stable against radiative corrections and we assume that Eq. (3) holds at an energy scale where these soft masses are given as the boundary conditions of the renormalization group equations.

In this model, the R-parity violating terms are parameterized by

\footnotetext{
${ }^{1}$ Note that in the gravity-mediated-supersymmetry-breaking scheme, the lepton-Higgs universality in the soft terms may be violated through interactions relevant above the Grand-Unified scale [12] [13].
} 


$$
\begin{aligned}
& s_{3} \equiv \sin \theta_{3}=\frac{\sqrt{\mu_{1}^{2}+\mu_{2}^{2}+\mu_{3}^{2}}}{\sqrt{\mu_{1}^{2}+\mu_{2}^{2}+\mu_{3}^{2}+\mu^{2}}}, \\
& s_{2} \equiv \sin \theta_{2}=\frac{\sqrt{\mu_{1}^{2}+\mu_{2}^{2}}}{\sqrt{\mu_{1}^{2}+\mu_{2}^{2}+\mu_{3}^{2}}}, \\
& s_{1} \equiv \sin \theta_{1}=\frac{\mu_{1}}{\sqrt{\mu_{1}^{2}+\mu_{2}^{2}}}
\end{aligned}
$$

Here, for simplicity, we have taken $\mu$ and $\mu_{i}$ to be real.

With (3) it is convenient to define a new basis of the Higgs and lepton supermultiplets in which the all bilinear mixing terms between the Higgs and leptons in the superpotential are absent:

$$
\left(\begin{array}{c}
H_{D}^{\prime} \\
L_{3}^{\prime} \\
L_{2}^{\prime} \\
L_{1}^{\prime}
\end{array}\right)=\left(\begin{array}{cccc}
c_{3} & s_{3} & 0 & 0 \\
-s_{3} & c_{3} & 0 & 0 \\
0 & 0 & 1 & 0 \\
0 & 0 & 0 & 1
\end{array}\right)\left(\begin{array}{cccc}
1 & 0 & 0 & 0 \\
0 & c_{2} & s_{2} & 0 \\
0 & -s_{2} & c_{2} & 0 \\
0 & 0 & 0 & 1
\end{array}\right)\left(\begin{array}{cccc}
1 & 0 & 0 & 0 \\
0 & 1 & 0 & 0 \\
0 & 0 & c_{1} & s_{1} \\
0 & 0 & -s_{1} & c_{1}
\end{array}\right)\left(\begin{array}{c}
H_{D} \\
L_{\tau} \\
L_{\mu} \\
L_{e}
\end{array}\right) .
$$

We can neglect the Yukawa couplings for the first and second generations. This approximation yields a global $U(1)$ symmetry, resulting in one massless neutrino $L_{1}^{\prime}=c_{1} L_{\mu}-s_{1} L_{e}$.

The rotation to take the new basis gives rise to R-parity violating trilinear couplings in the superpotential as well as the softly SUSY breaking terms in the scalar potential. For example a Yukawa coupling for bottom quark is written

$$
Y_{b} Q_{3} H_{D} D_{3}^{c}=Y_{b} Q_{3}\left(c_{3} H_{D}^{\prime}-s_{3} L_{3}^{\prime}\right) D_{3}^{c}
$$

Similarly a Yukawa coupling $Y_{\tau} L_{3} H_{D} \tau^{c}$ now reads

$$
\begin{aligned}
& Y_{\tau}\left(c_{2} s_{3} H_{D}^{\prime}+c_{2} c_{3} L_{3}^{\prime}-s_{2} L_{2}^{\prime}\right)\left(c_{3} H_{D}^{\prime}-s_{3} L_{3}^{\prime}\right) B^{c} \\
= & s_{2} s_{3} Y_{\tau} L_{2}^{\prime} L_{3}^{\prime} \tau^{c}+Y_{\tau} c_{2} L_{3}^{\prime} H_{D} \tau^{c}-Y_{\tau} s_{2} c_{3} L_{2}^{\prime} H_{D}^{\prime} \tau^{c} \\
\simeq & Y_{\tau} L_{\tau} s_{3}\left(s_{2}\left(c_{1} L_{\mu}+s_{1} L_{e}\right)\right) \tau^{c}+Y_{\tau} L_{\tau} H_{D}^{\prime} \tau_{c}
\end{aligned}
$$

Note that as far as $s_{3}$ is small, the old basis in Eq. (1) approximates a mass eigen basis for charged leptons. On the other hand, the new basis with prime gives in good approximation a mass eigen basis for neutrinos, as we will see shortly. 
As we mentioned before, radiative corrections violate the relation (3), resulting in vacuum expectation values(VEVs) of sneutrinos. An analysis shows that $\tilde{\nu}_{3}^{\prime}$ and $\tilde{\nu}_{2}^{\prime}$ develop VEVs. In fact the renormalization group effects induce terms of the form [15]

$$
\Delta B_{3} L_{3}^{\prime} H_{U}+\Delta m_{H L_{3}}^{2} L_{3}^{\prime} H_{D}^{\prime \dagger}+\Delta B_{2} L_{2}^{\prime} H_{U}+\Delta m_{H L_{2}}^{2} L_{2}^{\prime} H_{D}^{\prime \dagger}
$$

which gives the following sneutrino VEVs

$$
\begin{aligned}
\left\langle\tilde{\nu}_{i}^{\prime}\right\rangle & =\frac{\Delta B_{i} \tan \beta+\Delta m_{H L_{i}}^{2}}{m_{L_{i}}^{2}-\frac{1}{2} m_{Z}^{2} \cos 2 \beta}\left\langle H_{D}^{\prime}\right\rangle \quad(i=3,2) \\
\left\langle\tilde{\nu}_{1}^{\prime}\right\rangle & =0 .
\end{aligned}
$$

Note that $\Delta B_{3}$ and $\Delta m_{H L_{3}}^{2}$ are proportional to the bottom Yukawa coupling squared, while $\Delta B_{2}$ and $\Delta m_{H L_{2}}^{2}$ are proportional to the tau Yukawa coupling squared, and thus the ratio

$$
\tan \phi=\frac{\left\langle\tilde{\nu}_{2}^{\prime}\right\rangle}{\left\langle\tilde{\nu}_{3}^{\prime}\right\rangle}
$$

is generally small.

The non-vanishing VEV for the sneutrino induces mixing between the neutrino and the neutralinos, giving rise to a small mass for it due to the see-saw mechanism. Here it is useful to define a new basis

$$
\nu_{3}^{\prime \prime}=\nu_{3}^{\prime} \cos \phi+\nu_{2}^{\prime} \sin \phi, \nu_{2}^{\prime \prime}=\nu_{2}^{\prime} \cos \phi-\nu_{3}^{\prime} \sin \phi, \nu_{1}^{\prime \prime}=\nu_{1}^{\prime} .
$$

Then $\nu_{3}^{\prime \prime}$ acquires a tree-level mass

$$
m_{\text {tree }}=\frac{1}{2} g_{Z}^{2} v_{\nu}^{2} \sum_{i}\left(c_{W} O_{W \tilde{\chi}_{i}^{0}}-s_{W} O_{B \tilde{\chi}_{i}^{0}}\right)^{2} \frac{1}{m_{\tilde{\chi}_{i}^{0}}} .
$$

Here $m_{\tilde{\chi}_{i}^{0}}(i=1, \cdots, 4)$ represent neutralino masses and $O_{B \tilde{\chi}_{i}^{0}}$ and $O_{W \tilde{\chi}_{i}^{0}}$ stand for the contamination in $\tilde{\chi}_{i}^{0}$ of Bino and Wino components, respectively. Also $v_{\nu}=\sqrt{\left\langle\tilde{\nu}_{3}^{\prime}\right\rangle^{2}+\left\langle\tilde{\nu}_{2}^{\prime}\right\rangle^{2}}$.

The neutrinos acquire masses also at the one-loop level. The mass matrix induced from loop diagram contribution are diagonal for the $\nu_{i}^{\prime}$ basis. Relevant diagrams we should consider here are those which include the trilinear R-parity violating couplings. A one-loop diagram including scalar bottom quarks induces a following mass for $\nu_{3}^{\prime}$ : 


$$
m_{\nu_{3}^{\prime}}^{\text {loop }}=\frac{\left(Y_{b}^{2} s_{3}\right)^{2} m_{b}}{16 \pi^{2}} \frac{m_{\tilde{b}: L R}^{2}}{m_{\tilde{b} 1}^{2}-m_{\tilde{b} 2}^{2}} \log \frac{m_{\tilde{b} 1}^{2}}{m_{\tilde{b} 2}^{2}}
$$

Here $m_{\tilde{b}: L R}^{2}$ denotes a left-right mixing mass squared in the sbottom sector, and $m_{\tilde{b}_{1}}$ and $m_{\tilde{b}_{2}}$ are the eigenvalues of the scalar bottom quarks. Similarly a one-loop diagram involving scalar tau leptons gives a mass for $\nu_{2}^{\prime}$

$$
m_{\nu_{2}^{\prime}}^{\text {loop }}=\frac{\left(Y_{\tau}^{2} s_{3} s_{2}\right)^{2} m_{\tau}}{16 \pi^{2}} \frac{m_{\tilde{\tau}: L R}^{2}}{m_{\tilde{\tau} 1}^{2}-m_{\tilde{\tau} 2}^{2}} \log \frac{m_{\tilde{\tau} 1}^{2}}{m_{\tilde{\tau} 2}^{2}}
$$

Generically the latter contribution gives a dominant contribution to the mass of the second heaviest neutrino. The other neutrino remains massless in the approximation that the Yukawa couplings for the first two generations are set to be zero.

Summarizing, the mass matrix is written in the $\nu_{i}^{\prime \prime}$ basis

$$
m=\left(\begin{array}{ccc}
m_{\text {tree }}+m_{\nu_{3}^{\prime}}^{\text {loop }} \cos ^{2} \phi+m_{\nu_{2}^{\prime}}^{\text {loop }} \sin ^{2} \phi & \left(m_{\nu_{3}^{\prime}}^{\text {loop }}-m_{\nu_{2}^{\prime}}^{\text {loop }}\right) \sin \phi \cos \phi & 0 \\
\left(m_{\nu_{3}^{\prime}}^{\text {loop }}-m_{\nu_{2}^{\prime}}^{\text {loop }}\right) \sin \phi \cos \phi & m_{\nu_{3}^{\prime}}^{\text {loop }} \sin ^{2} \phi+m_{\nu_{2}^{\prime}}^{\text {loop }} \cos ^{2} \phi & 0 \\
0 & 0 & 0
\end{array}\right)
$$

When diagonalizing this matrix, the mixing angle for $\left(\nu_{3}^{\prime}, \nu_{2}^{\prime}\right)$ plane is

$$
\tan 2 \delta=\frac{2\left(m_{\nu_{3}^{\prime}}^{\text {loop }}-m_{\nu_{2}^{\prime}}^{\text {loop }}\right) \sin \phi \cos \phi}{\left(m_{\text {tree }}+\left(m_{\nu_{3}^{\prime}}^{\text {loop }}-m_{\nu_{2}^{\prime}}^{\text {loop }}\right) \cos 2 \phi\right)} \simeq \frac{2 m_{\nu_{3}^{\prime}}^{\text {loop }} \sin \phi \cos \phi}{m_{\text {tree }}} \ll 1
$$

Because the tree-level mass is generically larger than the one-loop corrections, we find that the $\nu_{i}^{\prime \prime}$ basis is in a good approximation a mass eigenstate.

Thus the mixing matrix of the neutrino sector, the MNS matrix [11, becomes

$$
\begin{aligned}
U_{i \alpha} & =\left(\begin{array}{lll}
U_{\tau 3} & U_{\tau 2} & U_{\tau 1} \\
U_{\mu 3} & U_{\mu 2} & U_{\mu 1} \\
U_{e 3} & U_{e 2} & U_{e 1}
\end{array}\right) \\
& =\left(\begin{array}{ccc}
1 & 0 & 0 \\
0 & c_{1} & -s_{1} \\
0 & s_{1} & c_{1}
\end{array}\right)\left(\begin{array}{ccc}
c_{2} & -s_{2} & 0 \\
s_{2} & c_{2} & 0 \\
0 & 0 & 1
\end{array}\right)\left(\begin{array}{ccc}
c_{\phi+\delta} & -s_{\phi+\delta} & 0 \\
s_{\phi+\delta} & c_{\phi+\delta} & 0 \\
0 & 0 & 1
\end{array}\right)=\left(\begin{array}{ccc}
c_{\theta} & -s_{\theta} & 0 \\
-c_{1} s_{\theta} & c_{1} c_{\theta} & -s_{1} \\
s_{1} s_{\theta} & s_{1} c_{\theta} & c_{1}
\end{array}\right)
\end{aligned}
$$


with $\theta=\theta_{2}+\phi+\delta$. Here $i$ and $\alpha$ denote the weak current basis and the mass eigen basis, respectively. Note that the MNS matrix obtained is of a very restrictive form with only two angles.

Now we would like to determine the mixing angles by using experimental data. Here we assume the hierarchical mass structure, namely $m_{3} \gg m_{2} \gg m_{1}$ so that $\Delta m_{32}^{2} \simeq \Delta m_{31}^{2} \gg$ $\Delta m_{21}^{2}$, as will be naturally realized in the model we are considering.

Let us first consider the atmospheric neutrino anomaly, which is explained by an oscillation $\nu_{\mu}-\nu_{\tau}$. The transition probability is

$$
\begin{aligned}
P\left(\nu_{\mu} \rightarrow \nu_{\tau}\right) & \simeq 4\left|U_{\mu 3}\right|^{2}\left|U_{\tau 3}\right|^{2} \sin ^{2} \frac{\Delta m_{32}^{2}}{4 E} L \\
& =4 c_{1}^{2} s_{\theta}^{2} c_{\theta}^{2} \sin ^{2} \frac{\Delta m_{32}^{2}}{4 E} L
\end{aligned}
$$

A recent analysis of the atmospheric neutrino at SuperKamiokande [17 indicates the following ranges at 90\% CL:

$$
\begin{aligned}
\Delta m_{32}^{2} & =(2-6) \times 10^{-3} \mathrm{eV}^{2} \\
4 c_{1}^{2} s_{\theta}^{2} c_{\theta}^{2} & >0.85 .
\end{aligned}
$$

On the other hand, the CHOOZ experiment 9] gives a bound on the mixing angle $U_{e 3}=s_{1} s_{\theta}$ to be

$$
4 s_{1}^{2} s_{\theta}^{2}\left(1-s_{1}^{2} s_{\theta}^{2}\right)<0.2
$$

for the mass range suggested by the atmospheric neutrino problem.

Combining Eq. (20) with Eq. (21), one finds that the $s_{1}$ has to be small. This non-trivial relation among $U_{e 3}, U_{\mu 3}$ and $U_{\tau 3}$ in the MNS matrix restricts patterns of the neutrino oscillations. In fact, for small $U_{e 3}$ implied by small $s_{1}$, the three flavor solar neutrino oscillation is well approximated by the usual two flavor oscillation solutions, namely the small angle MSW, the large angle MSW, and the vacuum oscillation [10. However, the latter two are excluded because the mixing angle involves $s_{1}$. This argument enables us to single out, in this model, the small angle MSW solution as the only possible explanation in terms of the 
neutrino oscillations to the solar neutrino problem. This is one of the main results of our paper.

For the small angle MSW solution, a recent result for the solar neutrino requires [2] $4 c_{\theta}^{2} s_{1}^{2} c_{1}^{2} \simeq(0.3-1) \times 10^{-2}$, which leads to

$$
s_{1} \simeq 0.04-0.07
$$

We shall next discuss the masses of the neutrinos. In general, the loop-level mass is suppressed compared to the tree-level mass simply due to the loop factors, giving a huge hierarchy among the neutrino masses. The atmospheric neutrino indicates the mass of the heaviest neutrino in the range of Eq. (19). The small angle MSW solution to the solar neutrino requires [2]

$$
\Delta m_{\mathrm{SMSW}}^{2} \simeq(0.4-1) \times 10^{-5} \mathrm{eV}^{2}
$$

To explain the atmospheric neutrino anomaly and the solar neutrino problem simultaneously in this framework of the R-parity violation, one has to make the tree-level mass relatively small, which requires a partial cancellation in the $\Delta B_{3} \tan \beta+\Delta m_{H L_{3}}^{2}$ term.

In the following, we shall demonstrate this fine tuning in the context of the GMSB [18,19]. Similar conclusions will be obtained for other mechanisms of supersymmetry-breaking mediation, such as the gravity mediation. In a simple GMSB model where the messenger quark belongs to a pair of 5 and $\overline{5}$ representations, the masses of squarks and gauginos are characterized by one parameter, the effective SUSY breaking scale $\Lambda$. The universal trilinear $A$ term as well as the universal $B$ term are assumed to be generated. All these parameters are given at some energy scale, which we identify rather arbitrarily with $\Lambda\left(=10^{5} \mathrm{GeV}\right)$ in the following analysis. We also introduce the supersymmetric $\mu$ term and $\mu_{i}(i=1,2,3)$ as independent parameters. Given $\tan \beta$, defined as the ratio of the two VEVs of the neutral Higgs bosons, the conditions of the radiative electroweak symmetry breaking fix the values of $\mu$ and $B$. The value $s_{3}$ is determined so that the heaviest neutrino mass lies in the region suggested by the atmospheric neutrino anomaly. Here we take its representative value to 
be $m_{\nu_{3}}=0.06 \mathrm{eV}$. As was explained earlier the other parameters $s_{2}$ and $s_{1}$ characterize the neutrino mixing angles.

In Figs. 1 and 2, we plot contours of the ratio of the two neutrino masses, $R=m_{\nu_{2}} / m_{\nu_{3}}$, in the $\tan \beta-A$ plane. Fig. 1 represents the case where $\mu<0$ and Fig. 2 corresponds to the case of $\mu>0$. One finds that the preferred ratio $R \simeq 0.03-0.07$ for the MSW solution to the solar neutrino is realized in small regions of the parameter space. We checked numerically that in these regions a cancellation among the two contributions to the VEV of the sneutrino indeed occurs.

We also examined the value of $s_{3}$. We found that the typical values of the $s_{3}$ is large, compared to a naive expectation of $\left(m_{\nu_{3}} / m_{W}\right)^{1 / 2} \sim 10^{-6}$. Especially in the region where the MSW solution is obtained, we checked that the value of $s_{3}$ can be $\sim 10^{-3}-10^{-5}$. These observations confirm that the alignment of the vacua works at least partially so that one does not need to assume a priori a very small $s_{3}$ to obtain small neutrino masses. Of course to simultaneously obtain the solutions of the atmospheric neutrino as well as the solar neutrino, we need the additional fine tuning to further suppress the sneutrino VEV.

Let us now turn to the question of how one can test this scenario at future colliders. When the R-parity is violated, the lightest superparticle (LSP) which is also the lightest R-parity odd particle is no longer stable, but decays to ordinary particles. In this paper we want to discuss patterns of the decays of the LSP. Here we assume that the LSP is a neutralino. Indeed in the model described above a bino-dominant neutralino becomes the LSP. For simplicity we also assume that it is heavier than the $\mathrm{W}$ boson. The LSP then decays to the $\mathrm{W}(\mathrm{Z})$ boson and a charged lepton (a neutrino) through the sneutrino VEV. Or it decays to three body decays via scalar lepton exchanges using the trilinear R-parity

\footnotetext{
${ }^{2}$ In the GMSB models, actually the LSP is the gravitino. However, one finds that the decay of the neutralino into the gravitino is negligibly small, and thus one can concentrate on the R-parity violating decay of the LSP among the MSSM superparticles.
} 
violating couplings. In either case, we checked that the decay length is short enough for the LSP to decay inside a detector for typical parameters discussed earlier. In Fig. 3, we exhibit the ratio of the partial width of the two body decay which involves the vector boson to that of the three body decays, $\Gamma\left(\tilde{\chi}_{1}^{0} \rightarrow\right.$ three leptons $) / \Gamma\left(\tilde{\chi}_{1}^{0} \rightarrow W l_{i}, Z \nu_{i}\right)$. We find that for the MSW solution the two body decays are generally dominant decay modes, though the decays through the trilinear Yukawa couplings may not be negligibly small.

Detailed analysis of the decays of the LSP may provide a test of the scenario we are discussing here. Let us illustrate this again when the LSP is bino-like. For the two body decay to a $\mathrm{W}$ boson and a charged lepton, the generation structure of the charged lepton directly reflects the mixing angles in the neutrino sector, and thus

$$
\frac{\Gamma\left(\tilde{\chi}_{1}^{0} \rightarrow \mu W\right)}{\Gamma\left(\tilde{\chi}_{1}^{0} \rightarrow \tau W\right)}=\frac{\left|U_{3 \mu}\right|^{2}}{\left|U_{3 \tau}\right|^{2}}=\tan ^{2} \theta .
$$

This will give information on the mixing angle of the atmospheric neutrino in this R-parity violation scenario. Note that the above relation is very generic, which is valid even when one relaxes the lepton-Higgs universality among the soft masses.

On the other hand, the three body decays occur through the following Yukawa couplings,

$$
Y_{\tau} s_{2} s_{3} c_{1}\left(\nu_{\tau} \mu-\nu_{\mu} \tau\right) \tau^{c}+Y_{\tau} s_{2} s_{3} s_{1}\left(\nu_{\tau} e-\nu_{e} \tau\right) \tau^{c}
$$

The neutralino LSP, which is in our case bino-like, couples most strongly to the right-handed stau and tau lepton and thus the decay occurs via the right-handed stau exchange and the final state will be either $\tau \tau \nu_{i}$ or $\tau \nu_{\tau} l_{i}$ where the generation suffix $i$ represents the first two generations. The latter final state is interesting because the tagging of the charged leptons in the first two generations will enable us to determine one parameter $s_{1}$. In fact we find

$$
\frac{\Gamma\left(\tilde{\chi}_{1}^{0} \rightarrow \tau \nu e\right)}{\Gamma\left(\tilde{\chi}_{1}^{0} \rightarrow \tau \nu \mu\right)}=\tan ^{2} \theta_{1} .
$$

This may provide us with an important information on our scenario where a large $s_{1}$ is excluded by the combined use of the CHOOZ experiment and the atmospheric neutrino.

To summarize, we have discussed the case of bilinear R-parity violation as a source of neutrino masses and mixing. We found that the resulting mixing matrix of the neutrinos 
has a very special pattern. This leads us to conclude that the large mixing angle solutions to the solar neutrino problem are ruled out when the CHOOZ result and the atmospheric neutrino data are combined together. Furthermore the relatively less hierarchical structure of the neutrino masses in this case are obtained if the soft SUSY breaking masses are suitably tuned to give small VEV for sneutrinos. Finally we argued that the analysis of the decays of the neutralino may reveal information on the mixing angles among the neutrinos. This scenario is testable at both neutrino oscillation experiments, e.g. SuperKamiokande [20], SNO [21], and KamLAND [22], and collider experiments in future.

\section{ACKNOWLEDGMENTS}

This work was supported in part by the Grant-in-Aid for Scientific Research from the Ministry of Education, Science, Sports, and Culture of Japan, on Priority Area 707

"Supersymmetry and Unified Theory of Elementary Particles", and by the Grant-in-Aid No.11640246 and No.98270. 


\section{REFERENCES}

[1] Super-Kamiokande Collaboration, Y. Fukuda et. al, hep-ex/9807003, Phys. Lett. B436 (1998) 133; Phys. Rev. Lett. 81 (1998) 1158.

[2] For recent results, see e.g. Y. Suzuki, talk given at International Symposium on Lepton and Photon Interactions at High Energies (Lepton-Photon'99), Stanford University, (August, 1999).

[3] For review, see H.P. Nilles, Phys. Rep. 110 (1984) 1; H.E. Haber and G.L. Kane, Phys. Rep. 117 (1985) 75.

[4] L.J. Hall and M. Suzuki, Nucl. Phys. B231 (1984 )419; I. Lee, Nucl. Phys. B246 (1984) 120; V. Barger, G.F. Giudice and T. Han, Phys. Rev. D40 (1989) 2987; K. Enqvist, A. Masiero and A. Riotto, Nucl. Phys. B373 (1992) 95; J.C. Romao and J.W.F. Valle, Nucl. Phys. B381 (1992) 87; F. Vissani and A.Yu. Smirnov, Nucl. Phys. B460 (1996) 37; K.S. Babu and R.N. Mohapatra, Phys. Rev. Lett. B384 (1996) 123; B. de Carlos and P.L. White, Phys. Rev. D54 (1996) 3424; A. Akeroyd, M.A. Diaz, J. Ferrandis, M.A. GarciaJareno and J.W.F. Valle, Nucl. Phys. B529 (1998) 3; E.J. Chun, S.K. Kang, C.W. Kim and U.W. Lee, Nucl. Phys. B544 (1999) 89; V. Bednvyakov, A. Faessler and S. Kovalenko, Phys. Lett. B442 (1998) 203; E.J. Chun and J.S. Lee, Phys. Rev. D60 (1999) 075006; K. Choi, E.J. Chun and K. Hwang, Phys. Rev. D60 (1999) 031301; R. Adhikari and G. Omanovic, Phys. Rev. D59 (1999) 073003; S. Rakshift, G. Bhattacharyya and A. Raychaudhuri, Phys. Rev. D59 (1999) 091701; B. Mukhopadhyaya, hep-ph/9907275; A. Abada and M. Losada, hep-ph/9908352.

[5] A. Joshipura and M. Nowakowski, Phys. Rev. D51 (1995) 2421; R. Hempfling, Nucl. Phys. B478 (1996) 3; T. Banks, Y. Grossman, E. Nardi and Y. Nir, Phys. Rev. D52 (1996) 5319; H.P. Nilles and N. Polonsky, Nucl. Phys. B484 (1997) 33; M.A. Diaz, J.C. Romao and J.W.F. Valle, Nucl. Phys. B524 (1998) 23; V. Bednyakov, A. Faessler and S. Kovalenko, Phys. Lett. B442 (1998) 203; A.S. Joshipura and S.K. Vempati, hep- 
ph/9808232; D.E. Kaplan and A.E.Nelson, hep-ph/9901254; A. Datta, B. Mukhopadhyaya and S. Roy, hep-ph/9905549; J.C. Romao, hep-ph/9907466; J.C. Romao, M.A. Diaz, M. Hirsch, W. Porod and J.W.F. Valle, hep-ph/9907499.

[6] T. Yanagida, in Proceedings of the Workshop on Unified Theories and Baryon number in the Universe, eds. O. Sawada and A. Sugamoto (KEK, Tsukuba, Japan, 1979); M. Gell-Mann, P. Ramond and R. Slansky, in supergravity, P. van Nieuwenhuizen and D. Freedman (eds), North-Holland, 1979.

[7] R. Godbole, P. Royand X. Tata, Nucl. Phys. B401 (1993)67; R. Hempfling, hepph/9702412; D.K. Ghosh and S. Raychaudhuri, Phys. Lett. B422 (1998) 187; B. Mukhopadhyaya, S. Roy and F. Vissani, Phys. Lett. B443 (1998) 191; M. Bisset, O.C.W. Kong, C. Macesanu and L.H. Orr, hep-ph/9811498; A. Akeroyd, M.A. Diaz and J.W.F. Valle, Phys. Lett. B441 (1998) 224; Y. Grossman and H.E. Haber, Phys. Rev. D59 (1999) 093008; S.Y. Choi, E.J. Chun, S.K. Kang and J.S. Lee, Phy. Rev. D60 (1999) 075002; D.K. Ghosh, R.M. Godbole and S. Raychaudhuri, hep-ph/9904233.

[8] OPAL Collaboration, G. Abbiendi et. al., hep-ex/9901037.

[9] CHOOZ Collaboration, M. Apollonio et. al., hep-ex/9907037.

[10] S.P. Mikheyev and A. Yu Smirnov, Phys. Lett. B200 (1988) 560; G. Fogli, E. Lisi and D. Monanino, Phys. Rev. D54 (1996) 2048; S. Bilenky, C. Giunti and C.W. Kim, Astropart. Phys. 4 (1996) 241; T. Teshima, T. Sakai and O. Inagaki, Int. J. Mod. Phys. A14 (1999) 1953; R. Barbieri, L.J. Hall, D. Smith, A. Strumia and N. Weiner, JHEP 12 (1998) 017. J.S. Kim and C.W. Kim, hep-ph/9909428

[11] L. Wolfenstein, Phys. Rev. D17 (1978) 2369; S.P. Mikheyev and A.Yu. Smirnov, Yad. Fiz. 42 (1985) 1441; Nuovo Cimento, C9 (1986) 17.

[12] Y. Kawamura, H. Murayama and M. Yamaguchi, Phys. Rev. D51 (1995) 1337.

[13] N. Polonsky and A. Pomarol, Phys. Rev. D51 (1995) 6532. 
[14] L.J. Hall and M. Suzuki, in Ref. [4]; R. Hempfling, in Ref. [5]; H.P. Nilles and N. Polonsky, in Ref. [5].

[15] V. Barger, M.S.Berger and P.Ohmann, Phys. Rev. D49 (1994)

[16] Z. Maki, M. Nakagawa and S. Sakata, Prog. Theor. Phys. 28 (1962) 870.

[17] T. Mann, talk given at International Symposium on Lepton and Photon Interactions at High Energies (Lepton-Photon'99), Stanford University, (August, 1999).

[18] M. Dine and A.E. Nelson, Phys. Rev. D48 (1993) 1277; M. Dine, A.E. Nelson and Y. Shirman, Phys. Rev. D51 (1995) 1362; M. Dine, A.E. Nelson. Y. Nir and Y. Shirman, Phys. Rev. D53 (1996) 2658.

[19] K.-I. Izawa, Y. Nomura, K. Tobe and T. Yanagida, Phys. Rev. D56 (1997) 2886; K.-I. Izawa, Y. Nomura and T. Yanagida, Phys. Lett. B452 (1999) 274.

[20] SuperKamiokande experiment; http://www-sk.icrr.u-tokyo.ac.jp/doc/sk/index.htm]

[21] SNO experiment; http://snodaq.phy.queensu.ca/SNO/sno.htm

[22] KamLAND experiment; http://www.awa.tohoku.ac.jp/html/KamLAND/index.html 


\section{FIGURES}

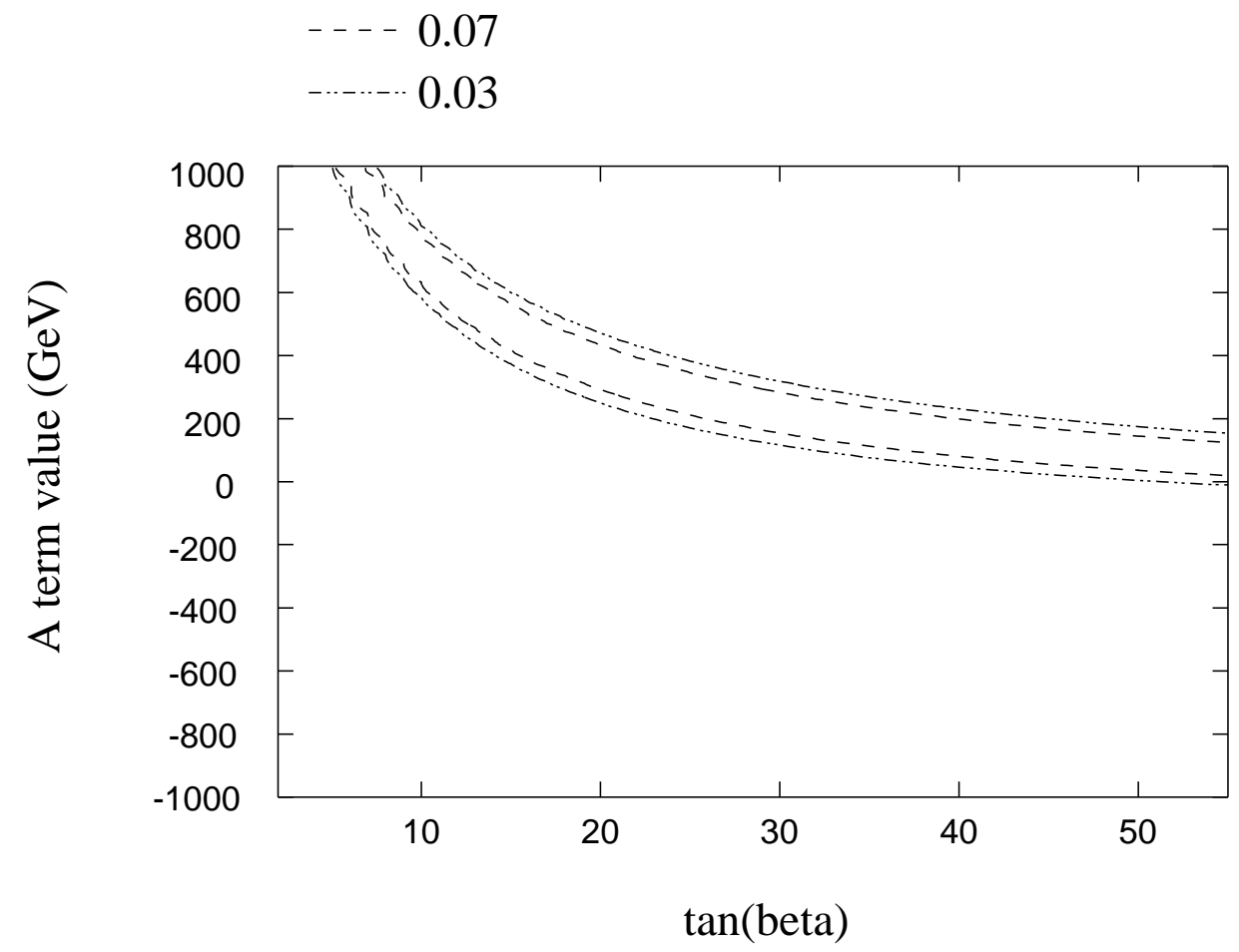

FIG. 1. Contour plot of $R=m_{\nu_{2}} / m_{\nu_{3}}$ on the trilinear coupling $A(\mathrm{GeV})$ - $\tan \beta$ plane for $\mu<0$. The dashed line corresponds to $R=0.07$ while the dot-dashed line corresponds to $R=0.03$.

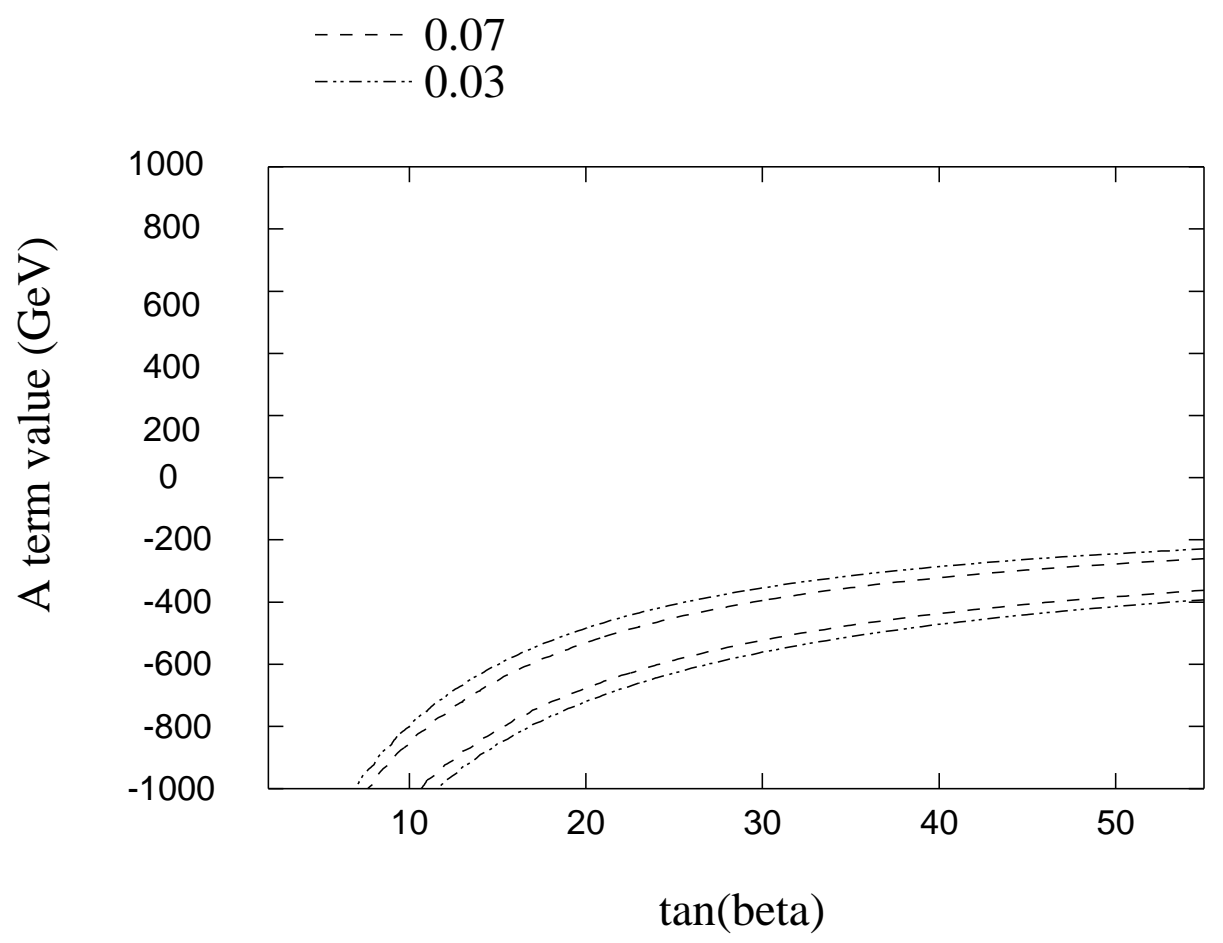

FIG. 2. The same figure as Fig. 1 except that $\mu>0$. 


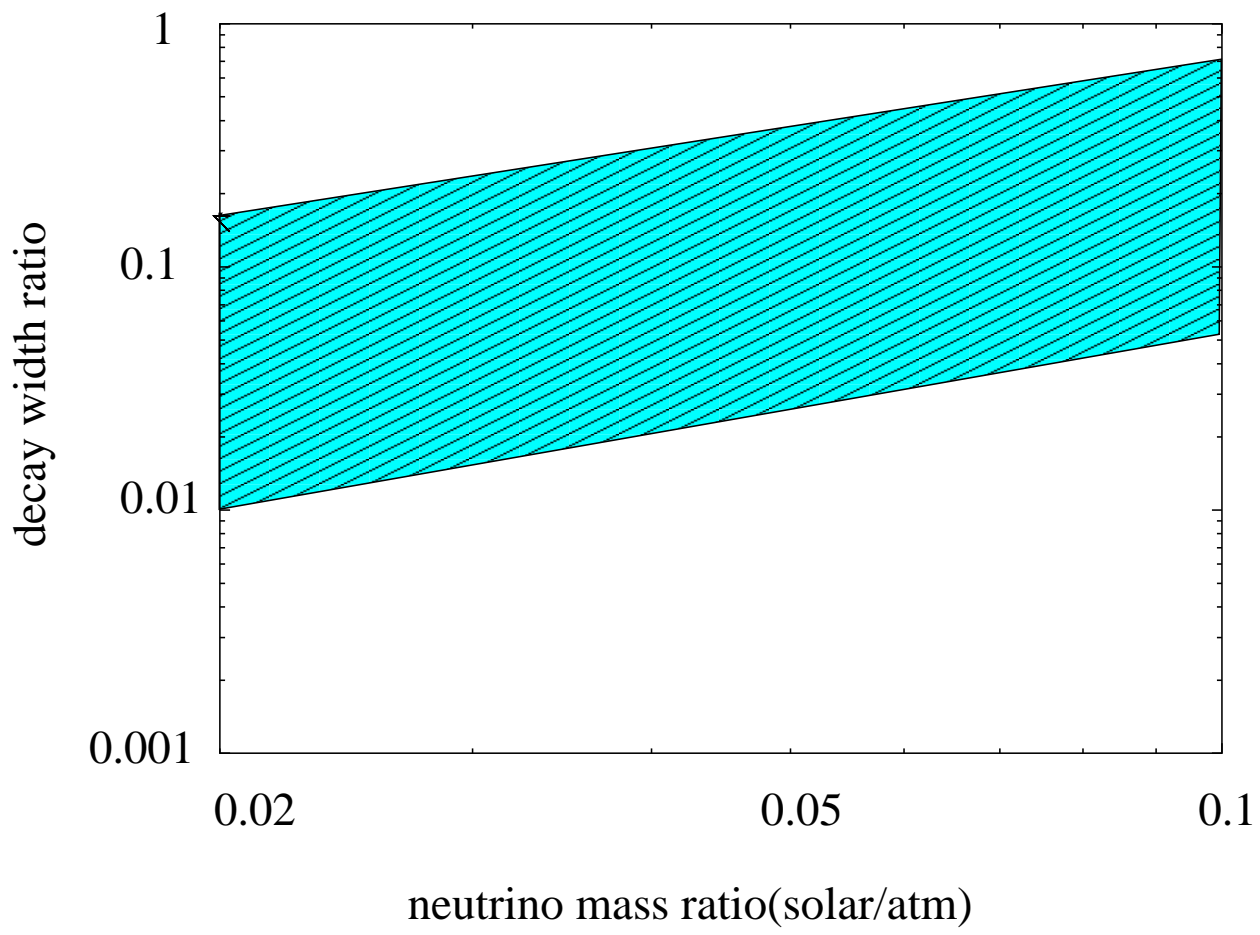

FIG. 3. The ratio of the decay widths $\Gamma\left(\tilde{\chi}_{1}^{0} \rightarrow\right.$ three leptons $) / \Gamma\left(\tilde{\chi}_{1}^{0} \rightarrow W l_{i}, Z \nu_{i}\right)$ versus the ratio of the neutrino masses $m_{\nu_{2}} / m_{\nu_{3}}$. The shaded region is obtained when $R=0.03-0.07$ is imposed. 